\title{
Cognitive decline and dementia in Down's syndrome
}

Authors:

Rosalyn Hithersay (first), UCL Division of Psychiatry, Gower Street, London, WC1E 6BT and LonDownS Consortium; r.hithersay@ucl.ac.uk

Sarah Hamburg, UCL Division of Psychiatry, Gower Street, London, WC1E 6BT and LonDownS

Consortium; s.hamburg@ucl.ac.uk

Bernice Knight, UCL Division of Psychiatry, Gower Street, London, WC1E 6BT;

bernice.knight.14@ucl.ac.uk

André Strydom (last and corresponding author), UCL Division of Psychiatry, Gower Street, London, WC1E 6BT and LonDownS Consortium; a.strydom@ucl.ac.uk

\begin{abstract}
Purpose of the review: Alzheimer's pathology is most likely universal in older individuals with Down syndrome (DS), due to having three copies of the amyloid precursor protein (APP) gene, resulting in amyloid-beta $(A \beta)$ plaque deposition. They are an important population in which to consider clinical trials of treatments to prevent or delay the development of dementia. However, assessment of subtler cognitive changes is challenging due to the presence of intellectual disability.

Recent findings: Recent research confirmed that older adults with DS often present with cognitive decline: more than $80 \%$ may experience dementia by age 65 . Efforts have been made to improve and validate neuropsychological assessment and to describe the relationship with comorbidities such as epilepsy and haemorrhagic stroke. There have also been advances in biomarkers such as neuroimaging using amyloid positron emission tomography.

Summary: Clinical trials of treatments, particularly in the presymptomatic phase of $A D$, are important to consider in individuals with DS given their high dementia burden, and may also serve as proof of concept for other forms of AD. However, further work is required to improve outcome measures and better understand the biomarkers of progression of pathology and their relationship with symptom development during the presymptomatic period.
\end{abstract}

\section{Key Words}

Down syndrome; Trisomy 21; Alzheimer's disease; Dementia; Cognitive Decline; 


\section{Key Points}

- The overall risk for Alzheimer's disease individuals with Down syndrome (DS) has been estimated to be more than $80 \%$ by age 65 ; the mean age at dementia diagnosis was 55.41 years.

- $\quad$ PET imaging using amyloid-beat binding compounds have demonstrated significant amyloid binding in several brain regions in all individuals with DS by age 50 .

- Haemorrhagic strokes and epilepsy are both more common in individuals with DS and dementia than in controls, though intracerebral haemorrhage may be less common in DS than in those with duplication APP (the chromosome 21 gene implicated in AD in DS) suggesting the presence of protective factors in DS.

- Several new test batteries for more detailed phenotyping of cognitive abilities in DS have been described, though further validation may be required for use as outcome measures in clinical trials.

- Several randomised treatment trials in DS populations has been published, showing some potential for EGCG to improve cognitive abilities before the onset of dementia in DS (though further trials are required), while Vitamin E to reduce cognitive decline was found to be ineffective. 


\section{Introduction}

Following significant increases in life expectancy, $40 \%$ of individuals with Down syndrome (DS; trisomy of chromosome 21) in the UK are now aged 40 years and older (1) with similar increases reported in the USA (2). Alzheimer's pathology is most likely universal in older individuals with DS, due to having three copies of the amyloid precursor protein (APP) gene, resulting in amyloid-beta $(A \beta)$ deposition in plaques (3). DS is therefore an important population in which to consider clinical trials of treatments to prevent or delay the development of dementia due to Alzheimer's disease (AD). However, assessment of more subtle cognitive changes in adults with DS is challenging due to the presence of intellectual disability (ID). This review will consider recent research on cognitive decline and dementia in people with DS, including epidemiology, clinical and neuropsychological assessment, comorbidities and advances in biomarkers such as neuroimaging.

\section{Epidemiology}

There is a sharp increase in dementia prevalence in older adults with DS, from less than $10 \%$ by age 49 , to more than $30 \%$ at 60 (3). As dementia is associated with high mortality rates, longitudinal data is required to estimate morbid risk. In a 14-year longitudinal study, the cumulative incidence for AD in women with DS was estimated to be more than $80 \%$ by age 65 ; the mean age at dementia diagnosis was 55.41 years $(S D=7.14)$ and median survival was 7 years after diagnosis (4).

Alexander et al. (5) compared age-specific incidence of previously recognised common cardiovascular, central nervous system, psychiatric, digestive and endocrine disorders in 6430 individuals with DS (3009 females and 3421 males) and 19,176 general population controls (8966 females and 10,210 males) using the United Kingdom Clinical Practice Research Datalink (CPRD) in a matched retrospective cohort study. They found a median age of 47 years for dementia diagnosis, with dementia incidence more than 90 times higher (IRR 92.1; 54.3-156.2; $P<0.001$ ) than in controls.

\section{Clinical assessment}

Diagnosing dementia in DS can be difficult, due to factors including variability of baseline cognitive level, heterogeneous presentation and lack of robust diagnostic tools. Sheehan et al. (6) used clinical data from memory assessments of 85 people with DS, of whom 64 (73.5\%) had an established diagnosis of dementia. Expert raters judged the cases as dementia or no dementia using their own clinical judgment and criteria from the International Classification of Disease (7) and Diagnostic Statistical Manual of Mental Disorders-IV-text revision (8). Clinicians' judgement was most accurate in identifying previously diagnosed cases (84.4\%), compared to $70.3 \%$ with ICD-10 criteria and $56.3 \%$ with DSM-IV-TR criteria. Over time, the proportion of cases meeting international criteria increased. The DSM-IV has recently been replaced by the DSM-5, which introduced a new term for dementia (major neurocognitive disorder) as well as a new diagnostic category for mild neurocognitive disorder, which may help to make earlier diagnoses in DS, though the validity of this diagnosis in the context of ID needs to be established (9).

Tsao et al. (10) used a cross-sectional study of 120 adults with DS without dementia aged 20 to 69 years, to examine the 'normal' ageing process. They found age-related deterioration in both cognitive and social adaptation skills with inter-individual variation, supporting the need for longitudinal assessment. 


\section{Co-morbidities}

Co-morbidities are common in DS, with well-established links between DS and congenital heart disease, epilepsy, hypothyroidism, visual and hearing impairments and depression $(5,11)$. The prevalence of most of these conditions does not change significantly in the presence of dementia (4), requiring clinicians to remain observant for their emergence irrespective of cognitive decline.

Alexander et al. (5) found incidence of epilepsy was elevated at all ages in DS, with a relative peak in adults 30 years and older (overall Incidence Relative Risk [IRR] 8.5; 7.3-9.8; $p<0.001$ and for age 30 years and older IRR 15.2). Epilepsy was significantly more common in DS where there was already a dementia diagnosis and occurred in more than $70 \%$ in a group with dementia (4). Epilepsy diagnosis was also a significant predictor of mortality $(p=0.002)$, with those with an epilepsy diagnosis being $6.39(2.02,20.19)$ times more likely to die than those without.

Sobey et al. (12) used population-level data to examine incident cardiovascular events in 4081 people with DS versus 16,324 age-matched controls. Haemorrhagic strokes were more common in people with DS than non-DS controls (risk ratio $[R R]=3.31,95 \%$ confidence interval $[\mathrm{Cl}] 1.95,5.60)$, as were ischaemic strokes ( $\mathrm{RR}=3.76,95 \% \mathrm{Cl} 2.39,5.92)$. Increased risk for ischaemic (but not haemorrhagic) stroke was substantially attenuated on adjustment for cardiovascular risk factors. Haemorrhagic stroke is most likely to be due to cerebral amyloid angiopathy (CAA) which results from the deposition of amyloid- $\beta$ within the walls of cerebral blood vessels, which can eventually lead to intracerebral haemorrhage. Buss et al. (13) reviewed epidemiological and neuropathological studies comparing a rare form of familial $A D$ caused by a duplication of the APP region on chromosome 21, with DS (three copies of chromosome 21). People with a familial duplication of APP appeared to have a higher frequency of intracerebral haemorrhage and possibly more extensive underlying vascular pathology. The authors concluded that Trisomy 21 may provide partial protection against the pro-haemorrhagic effects of APP duplication, and mechanisms related to amyloid- $\beta$ processing and clearance that may be responsible for differences in amyloid angiopathy require further examination. DS represents a unique opportunity to study the cerebrovascular features of aging and $A D$ in the context of protection against atherosclerosis and lower blood pressure but with significant CAA (14).

\section{Neuropsychology}

Floor effects are a particular concern when requiring tools that are sufficiently sensitive for tracking decline. Several widely used tests have recently been shown to be unsuitable for those with more severe ID or dementia, highlighting the need to develop more sophisticated neuropsychological tools for this population. However, object memory, semantic verbal fluency, the cued recall task, Tower of London, the CANTAB paired associate learning and simple reaction time tasks and the car and motorbike tracks of the NEPSY visuo-motor precision task have been highlighted as suitable for discriminating between those with and without dementia and tracking dementia related decline (15-19). The Raven's Coloured Progressive Matrices (20) also appear to show deterioration from age 40 onwards in dementiafree adults with DS (10). A closer look at the cued recall task originally described by Hartle (21) found both free and total recall scores discriminate age and dementia status in those with DS (22), however $\sim 35 \%$ of participants without dementia were excluded from the final sample for being unable to perform the task or cooperate, limiting the suitability of this test, particularly in those with lower functioning. 
Validation of the NIH toolbox Cognitive Battery for ID found Flanker inhibition/attention tasks, picture vocabulary, oral reading and picture sequence memory tasks were accessible to those with ID, whereas dimensional change card sort, pattern comparison and list sorting tasks showed large floor effects, limiting their suitability as trial outcome measures (23). However, this study focused on a young sample (mean DS age $=16$ ), including individuals with Fragile $X$ syndrome and idiopathic ID, thus the suitability of these tasks in older adults with DS remains unclear. The Rapid Assessment for Developmental Disabilities (RADD) battery (24) and the NeuroTrax Moderate to Severe Impairment Assessment Battery (25) have also been proposed as suitable tools for assessing cognitive function in those with DS, yet neither study reported full performance data.

A novel informant questionnaire addressing executive function, memory and language skills in DS was found to have high internal consistency, test-retest reliability and interrater reliability (26). This questionnaire could reliably discriminate between those with and without dementia, but showed no significant effects of age or gender, suggesting it could be useful for tracking cognitive decline, particularly where individuals are unable to engage with standard cognitive assessments.

\section{Dementia symptoms and presymptomatic decline in Down syndrome}

An ongoing debate is whether AD presents differently in DS compared to older adults from the general population. Dick et al. (27) compared neuropsychological profiles of higher-functioning DS individuals with dementia to individuals from the general population with advanced AD, adjusting for sex and functional impairment. They found similarities, suggesting the underlying pathology in both cases may have comparable effects on cognitive profiles.

Previous studies suggested that behavioural and psychological symptoms and executive dysfunction may precede memory impairment in DS dementia $(28,29)$. A recent cross-sectional study, however, suggested immediate memory impairment is one of the earliest signs of dementia in people with DS (30). Further longitudinal research is necessary to clarify the sequence of early changes in DS and their relationship to biomarkers such as neuroimaging.

It is important to remember that not all cases of cognitive decline in DS are the result of dementia. A rare phenomenon highlighted by recent research is that of childhood and adolescent "regression" or "disintegration", characterised by a sudden loss of daily living skills and cognitive decline. Worley et al. (31) summarized a case-series of what they termed DS disintegrative disorder, with results suggestive of a possible link with autoimmune function. A case study by Jacobs et al. (32) of a young adult male with DS experiencing similar deterioration highlights the diagnostic and treatment challenges these individuals face.

\section{Neuroimaging and other biomarkers}

Neuroimaging is a potential tool for monitoring biomarkers associated with progression from prodromal to symptomatic dementia in DS. Recent neuroimaging studies have used positron emission tomography (PET) scans with amyloid-beta $(A \beta)$ binding compounds to characterize amyloid deposition and its association with dementia onset and symptoms. Sabbagh et al. (33) used florbetapir PET imaging to assess mean standard uptake value ratios (SUVRs) as the primary outcome measure of fibrillar $A \beta$ burden in individuals with DS, DS with dementia and age-matched control subjects without DS. Significant group differences in mean SUVRs were reported, with uptake higher in DS individuals compared to controls, and greater still in those 
with DS and dementia.

Jennings et al. (34) also used florbetapir PET imaging with individuals with DS, demonstrating that binding was strongly correlated with age, but not significantly associated with cognitive decline. Annus et al. (35) used PET with Pittsburgh compound B (PiB; another A $\beta$ binding compound) in adults with DS, finding PiB binding first in the striatum around age 40 . By age 50, all had significant PiB binding in several brain regions. Age strongly predicted amyloid binding and PiB binding was also significantly associated with diagnostic status and history of cognitive decline. Taken together, these studies suggest amyloid-imaging techniques could be used to stage individuals with DS in the pre-symptomatic period according to amyloid positive status on scans, and to characterise and monitor changes associated with the progression of AD pathology longitudinally.

Additional neuroimaging studies include the investigation of EEG biomarkers, with Salem et al. (36) reporting an association between cognitive decline and theta slowing. Proton magnetic resonance spectroscopy has also been used with the putative glial biomarker myoinositol, which highlighted the possible existence of an overall higher glial inflammatory component in DS individuals (independent of dementia status) compared to control subjects (37).

\section{Biomarkers associated with cognitive functioning and decline in Down syndrome}

Plasma and CSF A $\beta$ levels are potential biomarkers for AD. In individuals with $D S$, worse performance in a semantic verbal fluency task, poorer communication skills and a higher Dementia Questionnaire for Persons with ID score were recently found to be associated with higher plasma $A \beta 42$ concentrations (38). Other AD biomarkers, including those related to inflammation and oxidative stress, are also of interest. Individuals with DS may have a unique neuroinflammatory state even before the onset of dementia, with implications for therapeutic intervention (39). Serum NGAL levels (Neutrophil Gelatinase-Associated Lipocalin, a newly identified neuroinflammatory constituent in $A D$ ) were shown to be significantly increased in DS subjects compared to those without $D S$, and positively associated with $A \beta 42$ and $A \beta(n 42)$ in $D S$ individuals with dementia and with $A \beta 40$ and $A \beta(n 40)$ in DS individuals without dementia (40). Declining plasma $A \beta 42$ and increasing proNGF (another inflammatory molecule) levels correlated with cognitive decline (41), while urinary isoprostane 8,12-iso-iPF2alpha, a marker of oxidative stress, was associated with cognitive decline in a longitudinal study of DS (42).

\section{Treatment trials}

Existing treatments, particularly acetylcholine esterase inhibitors, may be beneficial in individuals with DS who have dementia, but conclusive evidence for efficacy is lacking (43). Epigallocatechin-3-gallate (EGCG), a natural substance extracted from green tea, has been shown to inhibit DYRK1A (a kinase involved in Tau phosphorylation and implicated in AD in DS, as the DYRK1A gene is situated on chromosome 21) (44). De la Torre et al. (45) subsequently conducted a phase-2 randomized placebo-controlled trial of EGCG in young adults with DS ( $\mathrm{n}=$ 84 ) in combination with cognitive training to improve cognitive functioning. After 12 months, those in the treatment arm had significantly higher scores in measures of visual recognition memory, inhibitory control and adaptive behaviour, though the improvements were modest and further trials are required to determine longer-term safety and outcomes.

A randomized placebo-controlled trial of Vitamin $E$ to slow the progression of cognitive deterioration and dementia in individuals with DS was recently reported (46). Adults with DS older than 50 years $(n=337)$ were randomly assigned to receive either $1,000 \mathrm{IU}$ of vitamin $E$ 
orally twice daily or identical placebo for 3 years. There was no difference between drug and placebo on primary outcome (a brief praxis test), dementia rates, or secondary outcomes, with a trend towards higher rates of adverse events in those treated with vitamin $E$.

Cooper et al. (15) reported a small randomized placebo-controlled feasibility trial $(n=21)$ of simvastatin to prevent onset of dementia in older individuals with Down syndrome, using a memory for objects test as primary outcome. This test appeared to be sensitive to change, favouring the treatment, but the trial was too small to demonstrate benefit of the treatment.

\section{Conclusion}

Individuals with DS are a unique population for researching AD and developing clinical trials of drugs for primary prevention (to avoid amyloid overproduction and deposition) and secondary prevention (to avoid onset of dementia after amyloid deposition), due to the relatively predictable onset of dementia in these adults. Such trials may benefit individuals with DS given their high dementia burden and may also serve as proof of concept for other forms of AD, yet improvements in outcome measures and trial design are required (47). While recent research has improved our understanding of dementia in DS, further work is necessary to understand the progression of pathology and its relationship with the development of symptoms during the pre-symptomatic period of AD in DS. Prospective longitudinal studies offer great promise for our future understanding of $A D$ in $D S(18,48)$.

\section{Acknowledgements}

Thanks to Dr Tamara Al-Janabi for proof-reading and helping collate references

\section{Financial Support and Sponsorship}

None

\section{Conflicts of Interest}

AS has acted as an investigator for clinical trials sponsored by Roche Pharmaceuticals and consulted for Ono pharmaceuticals. He has been an adviser to the UK Down Syndrome Association and is an advisory board member of the LuMind Foundation (USA). No other authors have any competing interests to declare. 


\section{References}

1. Wu J, Morris JK. Trends in maternal age distribution and the live birth prevalence of Down's syndrome in England and Wales: 1938-2010. Eur J Hum Genet. 2013 Sep;21(9):943-7.

2. de Graaf G, Buckley F, Skotko BG. Estimation of the number of people with Down syndrome in the United States. Genet Med [Internet]. 2016 Sep 8 [cited 2016 Nov 25]; Available from: http://www.nature.com/gim/journal/vaop/ncurrent/full/gim2016127a.html

3. Wiseman FK, Al-Janabi T, Hardy J, Karmiloff-Smith A, Nizetic D, Tybulewicz VLJ, et al. A genetic cause of Alzheimer disease: mechanistic insights from Down syndrome. Nat Rev Neurosci. 2015 Sep;16(9):564-74.

4. **Mccarron M, Mccallion P, Reilly E, Mulryan N. A prospective 14-year longitudinal followup of dementia in persons with Down syndrome. Journal of Intellectual Disability Research. 2014;58(1):61-70.

5. Alexander M, Petri H, Ding Y, Wandel C, Khwaja O, Foskett N. Morbidity and medication in a large population of individuals with Down syndrome compared to the general population. Dev Med Child Neurol. 2016 Mar 1;58(3):246-54.

6. Sheehan R, Sinai A, Bass N, Blatchford P, Bohnen I, Bonell S, et al. Dementia diagnostic criteria in Down syndrome. Int J Geriatr Psychiatry. 2015 Aug 1;30(8):857-63.

7. World Health Organization. The ICD-10 Classification of Mental and Behavioural Disorders : Diagnostic Criteria for Research. Geneva: World Health Organization; 1993.

8. Association AP, others. Diagnostic and statistical manual of mental disorders DSM-IV-TR fourth edition (text revision). 2000;

9. Fletcher RJ, Barnhill J, McCarthy J, Strydom A. From DSM to DM-ID. Journal of Mental Health Research in Intellectual Disabilities. 2016 Jul 2;9(3):189-204.

10. Tsao R, Kindelberger C, Fréminville B, Touraine R, Gerald Bussy. Variability of the Aging Process in Dementia-Free Adults With Down Syndrome. American Journal on Intellectual and Developmental Disabilities. 2015;120(1):3-15.

11. Tassé MJ, Navas Macho P, Havercamp SM, Benson BA, Allain DC, Manickam K, et al. Psychiatric Conditions Prevalent Among Adults With Down Syndrome. Journal of Policy and Practice in Intellectual Disabilities. 2016 Jun 1;13(2):173-80.

12. Sobey CG, Judkins CP, Sundararajan V, Phan TG, Drummond GR, Srikanth VK. Risk of Major Cardiovascular Events in People with Down Syndrome. PLOS ONE. 2015 Sep 30;10(9):e0137093.

13. Buss L, Fisher E, Hardy J, Nizetic D, Groet J, Pulford L, et al. Intracerebral haemorrhage in Down syndrome: protected or predisposed? F1000Res [Internet]. 2016 May 12 [cited 2016 Nov 25];5. Available from: http://www.ncbi.nlm.nih.gov/pmc/articles/PMC4870990/

14. Wilcock DM, Schmitt FA, Head E. Cerebrovascular contributions to aging and Alzheimer's disease in Down syndrome. Biochimica et Biophysica Acta (BBA) - Molecular Basis of Disease. 2016 May;1862(5):909-14. 
15. Cooper S-A, Ademola T, Caslake M, Douglas E, Evans J, Greenlaw N, et al. Towards onset prevention of cognition decline in adults with Down syndrome (The TOP-COG study): A pilot randomised controlled trial. Trials. 2016;17:370.

16. de Sola S, de la Torre R, Sanchez-Benavides G, Benejam B, Cuenca-Royo A, del Hoyo L, et al. A new cognitive evaluation battery for Down syndrome and its relevance for clinical trials. Name: Frontiers in Psychology. 2015;6:708.

17. *Sinai A, Hassiotis A, Rantell K, Strydom A. Assessing Specific Cognitive Deficits Associated with Dementia in Older Adults with Down Syndrome: Use and Validity of the Arizona Cognitive Test Battery (ACTB). PLOS ONE. 2016 May 12;11(5):e0153917.

18. Startin CM, Hamburg S, Hithersay R, Davies A, Rodger E, Aggarwal N, et al. The LonDownS adult cognitive assessment to study cognitive abilities and decline in Down syndrome. Wellcome Open Research. 2016 Nov 15;1:11.

19. *Liogier d'Ardhuy X, Edgin JO, Bouis C, de Sola S, Goeldner C, Kishnani P, et al. Assessment of Cognitive Scales to Examine Memory, Executive Function and Language in Individuals with Down Syndrome: Implications of a 6-month Observational Study. Frontiers in Behavioral Neuroscience [Internet]. 2015 Nov 18 [cited 2015 Nov 26];9. Available from: http://journal.frontiersin.org/article/10.3389/fnbeh.2015.00300

20. Raven JC, Court JH. Raven's progressive matrices and vocabulary scales [Internet]. Oxford Psychologists Press Oxford, UK; 1998 [cited 2016 Nov 25]. Available from: http://www.vpsyche.com/doc/IQ/Raven-Vocabulary.doc

21. Hartle JT. Facilitation of recall with intralist cues: Time-dependent characteristics of cued recall. Memory \& Cognition. 1976 Sep 1;4(5):471-5.

22. Benejam B, Fortea J, Molina-López R, Videla S. Patterns of Performance on the Modified Cued Recall Test in Spanish Adults With Down Syndrome With and Without Dementia. American Journal on Intellectual and Developmental Disabilities. 2015 Oct 27;120(6):4819.

23. HessI D, Sansone SM, Berry-Kravis E, Riley K, Widaman KF, Abbeduto L, et al. The NIH Toolbox Cognitive Battery for intellectual disabilities: three preliminary studies and future directions. Journal of Neurodevelopmental Disorders. 2016;8:35.

24. Walsh DM, Doran E, Silverman W, Tournay A, Movsesyan N, Lott IT. Rapid assessment of cognitive function in down syndrome across intellectual level and dementia status. Journal of Intellectual Disability Research. 2015 Nov 1;59(11):1071-9.

25. Gutman M, Moskovic E, Jeret JS. Computerised cognitive testing of individuals with Down's syndrome and Alzheimer's disease. Journal of Intellectual Disability Research. 2016;60(2):179-81.

26. *Startin CM, Rodger E, Fodor-Wynne L, Hamburg S, Strydom A. Developing an Informant Questionnaire for Cognitive Abilities in Down Syndrome: The Cognitive Scale for Down Syndrome (CS-DS). PLOS ONE. 2016 May 6;11(5):e0154596. 
27. Dick MB, Doran E, Phelan M, Lott IT. Cognitive Profiles on the Severe Impairment Battery Are Similar in Alzheimer Disease and Down Syndrome With Dementia. Alzheimer Dis Assoc Disord. 2016 Sep;30(3):251-7.

28. Ball SL, Holland AJ, Treppner P, Watson PC, Huppert FA. Executive dysfunction and its association with personality and behaviour changes in the development of Alzheimer's disease in adults with Down syndrome and mild to moderate learning disabilities. British Journal of Clinical Psychology. 2008 Mar 1;47(1):1-29.

29. Ball SL, Holland AJ, Hon J, Huppert FA, Treppner P, Watson PC. Personality and behaviour changes mark the early stages of Alzheimer's disease in adults with Down's syndrome: findings from a prospective population-based study. Int J Geriat Psychiatry. 2006 Jul 1;21(7):661-73.

30. Blok JB, Scheirs JGM, Thijm NS. Personality and behavioural changes do not precede memory problems as possible signs of dementia in ageing people with Down syndrome. Int J Geriatr Psychiatry. 2016 Jan 1;n/a - n/a.

31. *Worley G, Crissman BG, Cadogan E, Milleson C, Adkins DW, Kishnani PS. Down Syndrome Disintegrative Disorder New-Onset Autistic Regression, Dementia, and Insomnia in Older Children and Adolescents With Down Syndrome. J Child Neurol. 2015 Aug 1;30(9):1147-52.

32. Jacobs J, Schwartz A, McDougle CJ, Skotko BG. Rapid clinical deterioration in an individual with Down syndrome. Am J Med Genet. 2016 Jul 1;170(7):1899-902.

33. ** Sabbagh MN, Chen K, Rogers J, Fleisher AS, Liebsack C, Bandy D, et al. Florbetapir PET, FDG PET, and MRI in Down syndrome individuals with and without Alzheimer's dementia. Alzheimer's \& Dementia. 2015 Aug;11(8):994-1004.

34. ** Jennings D, Seibyl J, Sabbagh M, Lai F, Hopkins W, Bullich S, et al. Age dependence of brain $\beta$-amyloid deposition in Down syndrome An [18F]florbetaben PET study. Neurology. 2015 Feb 3;84(5):500-7.

35. **Annus T, Wilson LR, Hong YT, Acosta-Cabronero J, Fryer TD, Cardenas-Blanco A, et al. The pattern of amyloid accumulation in the brains of adults with Down syndrome. Alzheimer's \& Dementia [Internet]. 2016 [cited 2015 Dec 10]; Available from: http://www.sciencedirect.com/science/article/pii/S1552526015026679

36. Salem LC, Sabers A, Kjaer TW, Musaeus C, Nielsen MN, Nielsen A-G, et al. Quantitative Electroencephalography as a Diagnostic Tool for Alzheimer's Dementia in Adults with Down Syndrome. Dement Geriatr Cogn Disord Extra. 2015 Oct 21;5(3):404-13.

37. Lin A-L, Powell D, Caban-Holt A, Jicha G, Robertson W, Gold BT, et al. 1H-MRS metabolites in adults with Down syndrome: Effects of dementia. Neurolmage: Clinical. 2016;11:728-35.

38. Hoyo LD, Xicota L, Sánchez-Benavides G, Cuenca-Royo A, de Sola S, Langohr K, et al. Semantic Verbal Fluency Pattern, Dementia Rating Scores and Adaptive Behavior Correlate With Plasma $A \beta 42$ Concentrations in Down Syndrome Young Adults. Front Behav Neurosci [Internet]. 2015 Nov 18 [cited 2016 Nov 25];9. Available from: http://www.ncbi.nlm.nih.gov/pmc/articles/PMC4649024/ 
39. Wilcock DM, Hurban J, Helman AM, Sudduth TL, McCarty KL, Beckett TL, et al. Down syndrome individuals with Alzheimer's disease have a distinct neuroinflammatory phenotype compared to sporadic Alzheimer's disease. Neurobiology of Aging. 2015 Sep;36(9):2468-74.

40. Naudé PJW, Dekker AD, Coppus AMW, Vermeiren Y, Eisel ULM, Duijn V, et al. Serum NGAL is Associated with Distinct Plasma Amyloid- $\beta$ Peptides According to the Clinical Diagnosis of Dementia in Down Syndrome. Journal of Alzheimer's Disease. 2015 Jan 1;45(3):733-43.

41. Iulita MF, Ower A, Barone C, Pentz R, Gubert P, Romano C, et al. An inflammatory and trophic disconnect biomarker profile revealed in Down syndrome plasma: Relation to cognitive decline and longitudinal evaluation. Alzheimer's \& Dementia. 2016 Nov;12(11):1132-48.

42. Zis $P$, McHugh $P$, McQuillin A, Praticò D, Dickinson $M$, Shende $S$, et al. Memory Decline in Down Syndrome and Its Relationship to iPF2alpha, a Urinary Marker of Oxidative Stress. PLOS ONE. 2014 Jun 5;9(6):e97709.

43. Eady N, Courtenay K, Strydom A. Pharmacological Management of Behavioral and Psychiatric Symptoms in Older Adults with Intellectual Disability. Drugs Aging. 2015 Feb 1;32(2):95-102.

44. De la Torre R, De Sola S, Pons M, Duchon A, de Lagran MM, Farré M, et al. Epigallocatechin3-gallate, a DYRK1A inhibitor, rescues cognitive deficits in Down syndrome mouse models and in humans. Mol Nutr Food Res. 2014 Feb 1;58(2):278-88.

45. * de la Torre R, de Sola S, Hernandez G, Farré M, Pujol J, Rodriguez J, et al. Safety and efficacy of cognitive training plus epigallocatechin-3-gallate in young adults with Down's syndrome (TESDAD): a double-blind, randomised, placebo-controlled, phase 2 trial. The Lancet Neurology. 2016 Jul;15(8):801-10.

46. **Sano M, Aisen PS, Andrews HF, Tsai W-Y, Lai F, Dalton AJ, et al. Vitamin E in aging persons with Down syndrome $A$ randomized, placebo-controlled clinical trial. Neurology. 2016 May 31;86(22):2071-6.

47. Rafii MS. Improving Memory and Cognition in Individuals with Down Syndrome. CNS Drugs. 2016 Jul 1;30(7):567-73.

48. Fortea J, Carmona-Iragui M, Fernandez S, Benejam B, Videla L, Alcolea D, et al. DOWN ALZHEIMER BARCELONA NEUROIMAGING INITIATIVE (DABNI): A PROSPECTIVE LONGITUDINAL BIOMARKER COHORT TO STUDY ALZHEIMER'S DISEASE IN DOWN SYNDROME. Alzheimer's \& Dementia. 2016 Jul;12(7):P380-1. 


\section{Key Papers to Highlight}

\section{4 ** McCarron et al. (2014)}

- Provides an estimate of the morbid risk/ cumulative incidence for dementia in older adults with Down syndrome - no other similar papers in last two years, so this is an important study to highlight

\section{*Liogier d'Ardhuy et al., (2015)}

- A 6-month observation study determining the effectiveness of several cognitive tools to use for future interventional clinical studies

*Sinai et al., (2016)

- This paper presents one of the first validations of the Arizona Cognitive Test Batteries in older individuals with DS, including those with dementia, highlighting tasks which work well and tasks which are unsuitable for measuring cognition in these adults

*Startin et al., (2016)

- This paper presents a novel informant questionnaire, the 'CS-DS', to assess cognitive abilities in individuals with DS at all levels of functioning, while also being able to distinguish between those who have and do not have a clinical diagnosis of dementia

\section{1 *Worley et al., (2015)}

- Paper is of special interest because it describes several cases of 'Down syndrome disintegrative disorder' i.e. regression/decline in children and adolescents with DS

33-35**Sabbagh et al., (2015), Jennings et al., (2015) and Annus et al., (2016)

- Three important studies that have successfully conducted PET imaging studies in adults with Down syndrome to look at $\beta$-amyloid deposition in vivo

$45 *$ de la Torre et al., (2016)

- A paper reporting a randomised clinical trial of EGCG in younger individuals with Down syndrome, with some evidence for an effect

$46 * *$ Sano et al., (2016)

- A randomised trial of vitamin E (as anti-oxidant) in older individuals with Down syndrome, demonstrating lack of efficacy 